\title{
EINIGE FRAGEN \\ DER AKTIONSART- UND ASPEKTFUNKTION IM SPRACHVERGLEICH
}

ABSTRACT: On the functions of ,, aktionsart" and aspect in language contrast

The contribution deals with selected questions of the interaction between the so called "lexical aspect" (the opposition between telicity and atelicity) and the grammatical aspect (or so called "viewpoint"-aspect, i.e. the opposition between perfectivity and imperfectivity) in the languages with and without the overtly encoded aspect. The striking point of the analysis is the "complexive" meaning of aspectual forms and constructions involving lexical atelicity by indicating durativity or iterativity, on the one hand, and grammatical perfectivity by indicating the complexive perspective of the verbal action on the other. This type of aspectuality was a special feature of verbal systems with the aorist category. My claim is, thus, that the contemporary English has a special grammatical form of the "complexive aorist", i.e. the form of Present Perfect Progressive. The Slavic languages encode this function by using the - unmarked - imperfective forms of the verbs, whereas German uses special means of encoding the very same function on the whole-clause level, such as adverbials or definite vs. indefinite or zero article.

KEYwORDS: viewpoint aspect; lexical aspect; aktionsart; complexivity; aorist

\section{Einleitende Bemerkungen und Zielsetzung}

Der Beitrag von Prof. Józef Darski zur Entwicklung der Theorie der Linguistik mit universaltypologischem Ansatz ist allgemein anerkannt, und seine Grundthesen wurden seit der Publikation seiner ersten Forschungsergebnisse zu diesem Themen- und Problemkreis mehrfach unter Beweis gestellt. Das komplexe, mehrstufige Modell einer universalistisch konzipierten Sprachdeskription von Józef Darski (vgl. u. v. a. Darski 1999; 2004; 2010; 2015) erfasst sämtliche Ebenen des Sprachsystems und weist eine makrolinguistisch basierte Hierarchie - vom Text zum Morphem und Phon - auf (vgl. Harris 1951). Die von Darski behandelten relevanten Distinktionen gelten sowohl für

Michail L. Kotin - Uniwersytet Zielonogórski, michailkotin1@gmail.com 
eine völlig neu konzipierte Einteilung von Wortklassen und Satzgliedern („Äußerungsgliedern") als auch für innovative Zugriffe auf die Analyse grammatischer Wortformen (also Feststellung der Nomenklatur der Grammeme, die er entsprechend als referentielle Wortstämme und syntaktische Exponenten bezeichnet, vgl. Darski 2004; 2010; 2015). Sein expliziter Rückgriff auf die Glossematik von Hjelmslev (1943) zeigt im Allgemeinen einen in jeder Hinsicht suffizienten Lösungsvorschlag bei der Suche nach einem konsequenten und angemessenen Beschreibungsmodell vom gesamten Sprachsystem mit universaltypologischem Grundansatz und Geltungsanspruch.

Der Verfasser des vorliegenden Beitrags hat in seinen früheren Arbeiten (vgl. Kotin 2008; 2012) bereits gezeigt, dass das ursprünglich synchron konzipierte linguistische Analysemodell von Józef Darski durchaus mutatis mutandis auf diachron angelegte Untersuchungen im Bereich der (historischen) Morphologie und Syntax übertragbar ist und dort ohne essentielle Einschränkungen verwendet werden kann - konkrete Beispiele dieser Anwendung sind die Arbeiten von Kotin (2012) sowie Woźnicka (2014).

Der vorliegende Aufsatz stellt den Versuch dar, an exemplarischen Fallbeispielen gewisse empirisch feststellbare und theoretisch begründbare kategoriale Affinitäten auf der Ebene komplexer Satzstrukturen zu demonstrieren. Dies wird vom Verfasser als eine bescheidene Ergänzung der im letzten Buch von Darski (Darski 2015) vorgenommenen komplexen Kontrastierung grammatischer Wortformen und Strukturen des Deutschen und des Polnischen verstanden. Zugleich wird versucht, darüber hinaus einige gemeinsame kategoriale Signale zu ermitteln, welche über Sprachkontrast hinausgehen und eher typologischer Natur sind.

Der Beitrag ist wie folgt aufgebaut: Das Unterkapitel 2 ist eine kurze theoretische Einführung in die sehr komplexe und viel diskutierte Problematik der Aspekt- bzw. Aktionsartfunktionen, wobei lediglich die für die weitere Darstellung unmittelbar relevanten Fragen aufgegriffen werden. Diese recht restriktive Selektion ist angesichts der äußerst umfangreichen und heute kaum überschaubaren Fachliteratur zu den Themen Aspekt und Aktionsarten notwendig. Im Unterkapitel 3 werden zunächst Fallbeispiele präsentiert und behandelt, die eine funktionale Ähnlichkeit ausdrucksseitig divergenter Entitäten und Konstruktionen in zu vergleichenden Sprachsystemen demonstrieren. Es handelt sich vor allem um kategoriale Äquivalente für grammatisch kodierte Aspektoppositionen der Slavia (Polnisch und Russisch) in den germanischen Sprachen Deutsch und Englisch, welche keine bzw. keine mit der Slavia vergleichbaren Kodierungsformen der Aspektfunktion aufweisen. Zugleich wird gezeigt, dass das Englische aspektuale bzw. aspektbezogene Oppositionen viel systematischer als das Deutsche und in einigen spezifischen Kategorialbereichen sogar als die Slavia kodiert. Ferner wird im abschließenden Unterkapitel 4 versucht, die ermittelten Affinitäten auf einen gemeinsamen „kategorialen Nenner“ zu bringen und die allgemeine Bilanz der vorgenommenen Analyse wird gezogen.

Die Grundhypothese ergibt sich weitgehend aus den theoretischen Überlegungen Darskis zur Universalität grammatischer Grundfunktionen bei Divergenz ihrer Kodie- 
rungsformen sowie aus den Studien zur typologisch ausgerichteten Grammatik von Comrie (1976), Bybee/Dahl (1989), Abraham/Janssen (1989), Abraham/Leiss (2012) mit universaltypologisch konzipiertem theoretischem Anspruch. Die Analyse ist synchron-kontrastiv und sprachtypologisch angelegt, wobei sich der Verfasser durchaus der Tatsache bewusst ist, dass eine sprachtypologische Geltung der Ergebnisse seiner Analyse angesichts der geringen Anzahl der zu analysierenden Sprachen und ihrer Zugehörigkeit zur Sprachfamilie der Indogermania äußerst beschränkt ist. Darüber hinaus soll ausdrücklich betont werden, dass die hier diskutierten Beispiele eine allgemeine Vorlage bzw. Folie für weitere, korpusgestützte Studien bilden, welche die hier aufgestellten Thesen am umfangreichen und statistisch angemessen ausgewerteten Belegmaterial überprüfen sollten.

\section{Aspekt- und Aktionsartfunktionen}

Die Unterscheidung zwischen Aspekt und Aktionsart wird in der einschlägigen Literatur am häufigsten als eine kategoriale Basisdistinktion behandelt (vgl. Klein 1994; 1995: 669-672; Maslov 2004: 165ff.; Eckhof/Haug 2015: 190-193). Es wird dabei grundsätzlich zwischen dem sog. ,lexikalischen“ Aspekt und dem grammatischen, oder „viewpoint"“-Aspekt unterschieden (vgl. Klein 1994; Schwall 1991: 92f.). Während der erstere eine kategoriale Komponente der jeweiligen lexikalischen Verbbedeutung ist, ist der letztere eine morphologisch kodierte funktionale Opposition zwischen den aspektualen Lesarten desselben Verbalstammes.

Während nun der grammatische Aspekt eine deutlich ausgeprägte kategoriale Opposition zwischen Imperfektivität und Perfektivität indiziert, gibt es bei der kategorialen Einordnung der Aktionsarten keine Übereinstimmung. Einerseits liefern die einschlägigen Arbeiten mehr oder weniger subtile Klassifizierungen der Aktionsarten mit Haupt- und Untertypen sowie Misch- und Übergangsformen (vgl. Isačenko 1960; Forsyth 1972; Kątny 1994; Bondarko 1995; Czarnecki 1998 u. a.); andererseits werden Verallgemeinerungsvorschläge gemacht, die in ihrer kategorialen Binarität weit über die lexikalische Determination der Aktionsartfunktionen hinauslaufen und eher an Oppositionen erinnern, die vielmehr für Grammeme als für kategoriale Seme lexikalischer Bedeutungen typisch sind (vgl. Dahl 1985: 73ff.). Zu den letzteren Zugriffen gehört ohne Zweifel die angesetzte kategoriale Distinktion zwischen den Merkmalen [telisch] vs. [atelisch] (vgl. Łazorczyk 2010: §2.3.2.; Eckhoff/Haug 2015: 190f.). Eckhoff/Haug (2015: 190f.) sehen den Grundunterschied zwischen telischen und atelischen Prädikaten darin, dass im ersten Fall das Ereignis auf seinen Abschluss gerichtet ist, während im letzteren Fall diese Ereignisrichtung fehlt.

Demgemäß kann nun zunächst eine essentielle kategoriale Korrelation zwischen der binären Opposition [telisch] vs. [atelisch] und den mehrgliedrigen Oppositionen diverser Aktionsartverben (Durativa, Iterativa, Habituativa, Ingressiva, Inchoativa, 
Resultativa, Finitiva, Saturativa und deren Unterarten, die in der Literatur zu Aktionsartsemantik beschrieben worden sind) festgestellt werden. Offenkundig ist hier die Verteilung recht eindeutig: Die atelischen Verben gehören nämlich der einen Gruppe der Aktionsarten (durativ, iterativ, habituell u. a.) an, während die telischen Verben die andere Gruppe (ingressiv-inchoative, resultativ-finitive u. a.) umfassen. So ist es klar, dass die beiden „Pole“ der ternären Sequenzen der Aktionsartverben bzw. -prädikate beginnen - dauern - enden, erblühen - blühen - verblühen, entstehen - bestehen verschwinden, geboren werden - leben - sterben $\mathrm{u}$. a. telisch, während die jeweils in der Mitte stehenden Verben/Prädikate atelisch sind.

Wie ist nun die Korrelation dieser kategorialen Anordnung der verbalen Aktionsarten zu dem grammatischen, also dem ,,viewpoint"-Aspekt zu bestimmen? Dieser Letztere ist im Unterschied zur Aktionsart ein Indikator der Fokussierung der Verbalhandlung, welche üblicherweise über Temporalität (als eine Art, ,interne Temporalität“ im Unterschied zur „externen“, durch Tempusformen bezeichneten Temporalität) definiert wird (vgl. Comrie 1976: 3: „,aspects are different ways of viewing the internal temporal constituency of a situation"), auch wenn, wie im Weiteren gezeigt wird, die Temporalität im Prinzip aus der Aspektdefinition ausgeschlossen werden kann (im Vorgriff darauf vgl. Leiss 1992: 47). Unter Bezugnahme auf ,interne“ Temporalität kann nun die Grundfunktion des imperfektiven Aspekts als Fokussierung des partiellen Zeitintervalls des Verlaufs des Verbalgeschehens definiert werden, während die Grundfunktion des perfektiven Aspekts in der Fokussierung der vollständigen Verlaufszeit des Verbalgeschehens besteht (vgl. Eckhoff/Haug 2015: 190), vgl. poln. robić - zrobić 'tun - (bis zu Ende) getan haben', jeść - zjeść 'essen - aufessen', czytać - przeczytać 'lesen - (bis zum Ende) gelesen haben', umierać - umrzeć 'im Sterben liegen - sterben' u. a. Diese Differenzierung gilt für alle Sprachen, die die Kategorie des sogenannten „Begrenzungsperfektivs“ (Bybee/Dahl 1989: 86) besitzen, darunter alle slawischen Sprachen, die baltischen Sprachen (Lettisch und Litauisch) sowie einige andere außerindoeuropäische Sprachen, z. B. Ungarisch, Georgisch, Margi und einige Sprachen Mikronesiens (Bybee/Dahl 1989: 86).

Bekanntlich werden die slawischen Sprachen als Paradebeispiel der Aspektsprachen behandelt - vor allem, weil sie einen extrem hohen Grad an Paradigmatisierung der Aspektformen haben (vgl. Jakobson 1932; Comrie 1976). Doch der „,slawische Aspekt" ist in vielerlei Hinsicht spezifisch, nach der Meinung einiger Forscher sogar „typologisch unüblich“ (Dahl 1985: 84) und nicht immer generell für „klassische“ Aspektvorstellungen geeignet. Eines der Probleme besteht nämlich in einer relativen Unabhängigkeit des ,slawischen“ Aspekts vom Tempus und der Zeitreferenz. Weitere Faktoren sind der derivationelle (und nicht der flexivische) Charakter der Aspektkodierung sowie die engste Verbundenheit des Aspekts mit der Telizität: Perfektive Verben haben nämlich nur telische Lesarten (Dahl 1985: 84f.), was für andere Aspektsprachen mit dem sogenannten „Begrenzungsperfektiv“ nicht gilt. Diese drei Merkmale, die laut Dahl (1985: 84f.) den „slawischen“ Verbalaspekt „typologisch unüblich“ machen, erfordern eine eingehendere Erörterung. 
(i) Die relative Unabhängigkeit des Aspekts vom Tempus ist aus zweifacher Sicht kein sicheres Kriterium. Erstens ist sie eben nur „relativ“, d. h., es gibt genug Indizien für den engsten kategorialen Zusammenhang zwischen beiden grammatischen Kategorien. Das entscheidendste davon ist nämlich die prospektive Lesart der Präsensformen der slawischen Perfektiva, wodurch sie nur zwei Tempusstufen (Vergangenheit und Zukunft) kodieren können, während die Imperfektiva drei Tempora für die Bezeichnung der Vergangenheit, der Gegenwart und der Zukunft aufweisen. Zweitens ist die Bindung der Aspektfunktion an die Tempussemantik - auch indirekt verstanden - nicht zwingend notwendig. Wie Leiss (1992: 47) gezeigt hat, ist die Aspektdefinition als Kodierung der Opposition von Innen- vs. Außenperspektivierung des Verbalgeschehens auch ohne Heranziehen der Zeitreferenz suffizient. In diesem Fall fokussiert der imperfektive Aspekt die Position des Betrachters (der Origo) innerhalb des additiven Geschehens, während der perfektive Aspekt eine Betrachtung des (non-additiven, als ganzheitlich konzipierten) Geschehens von außen kodiert. Diese Sichtweise ermöglicht u. a. die Übertragung der Aspektbehandlung generell von der Wortform- auf die Satzebene, unter anderem unter Einbeziehung der Nominalphrase, was noch unten speziell gezeigt wird.

(ii) Der derivationelle Charakter der Aspektkodierung schwächt seine Grammatikalizität kaum ab, ebenso wenig, wie z. B. der ebenfalls derivationelle Charakter der Pluralbildung der Substantive im Deutschen im Unterschied zum flexivischen im Russischen oder im Polnischen die deutliche kategoriale Parallelität beider Oppositionsglieder der Numerusopposition des Substantivs zu schmälern vermag. Schließlich wird das germanische schwache Präteritum auch rein derivationell gebildet, was keineswegs von einer mangelhaften Grammatikalisierung dieser Tempusform zeugt. Zusätzlich muss hier ausdrücklich betont werden, dass gerade bei typologischen Studien eine explizite Gegenüberstellung von den Kodierungsformen für vergleichbare Kategorialfunktionen etwas kurios erscheint. Zugegeben ist allerdings die derivationelle Kodierungsform in einer ausgeprägt flektierenden Sprache (wie die Slavia) stets mit zusätzlichen Merkmalen verbunden, von denen wohl das wichtigste eine sich daraus ergebende stärkere „Lexikalizität“ der derivationell kodierten grammatischen Kategorie, ihre größere Affinität zur Wortsemantik ist, worauf u. a. Jarceva (1968: 30) bereits aufmerksam gemacht hat.

(iii)Die starke Verbundenheit der Aspektfunktion (imperfektiv : perfektiv) mit der Aktionsartfunktion (atelisch : telisch) ergibt sich wiederum weitgehend aus der unter (ii) behandelten derivationellen Kodierungsform beider Funktionen, oder, genauer gesagt, sie wird gerade durch die - historisch bekanntlich wesentlich jüngere - derivationelle Kodierungsform der Aspektfunktion im Slawischen besonders sichtbar gemacht.

Eine Vergleichsbasis für die adäquate kategoriale Zuordnung der Aspektfunktionen in deren Relation zur Telizität bzw. Atelizität können Sprachsysteme bieten, in denen beide Funktionen definitiv getrennt denotiert werden konnten. Dies kann verständli- 
cherweise nur in einem Vergangenheitstempus geschehen, wo Telizität und Atelizität temporal ,gleichstufig“ interpretierbar sind, wohingegen die formal gegenwartstemporalen Formen atelischer und telischer Verben prinzipiell unterschiedliche Zeitreferenz aufweisen, also respektive Gegenwarts- und Zukunftsbezug, vgl. poln. pisze 'schreibt': napisze 'wird geschrieben haben'. Ein gutes Beispiel ist hier das Altgriechische, insbesondere in der jüngsten, hellenistischen Zeit seiner Geschichte, als u. a. viele genuine Perfektformen weitgehend durch Aoristformen ersetzt wurden (vgl. schon Streitberg 5/61920: §298, Anm. 1 sowie Moulton 1906: 140; Fanning 1990: 299; Jones 2009: 224). Aber auch das Altkirchenslawische als gemeinsame Vorstufe in der Entwicklung der Slavia weist ein weitgehend mit dem Griechischen vergleichbares Bild auf, indem es ebenfalls neben dem Imperfekt den sehr stark funktional aufgefächerten Aorist besitzt (vgl. Dostál 1954: 15f.).

Interessant für diese Studie ist hier die Verwendung des Aorists in komplexiver und konfektiver Lesart. Beide indizieren nämlich Ereignisse, welche von der Aktionsart her atelisch und von der Aspektfunktion her perfektiv sind. Andauernde Handlungen oder Prozesse werden dabei aus der Sicht des (,grammatischen“) viewpoint-Aspekts als quasi abgeschlossen gedeutet, und zwar im Sinne einer Außenperspektivierung der additiven Aktionsartfunktion. Die komplexive Lesart des griechischen und altkirchenslawischen Aorists stellt ein Ereignis, welches nicht auf ein Ende gerichtet ist, als Intervall dar, bei welchem nicht die Dauer, sondern die zeitlichen Grenzen derselben das kategoriale Grundprofil bilden (vgl. Eckhoff/Haug 2015: 192). Der konfektive Aorist bezeichnet einen Prozess, der vor Eintritt eines anderen Ereignisses als abgeschlossen galt (vgl. Krasuchin 2007: 32):

(1) molišę pilata da prěbijoţ golěni ixъ (Joh. 19, 31, Codex Zographensis), sie baten Pilatus, dass er ihre Knie zerbrechen lässt.' (nach Eckhoff/Haug 2015: 210).

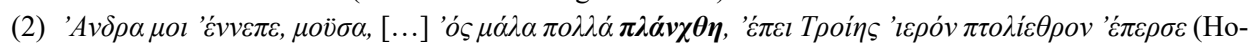
mer, Odys. I, 1-2), Einen Mann besinge mir, o Muse, [...], der viel gereist war, nachdem er Trojas starke Befestigung zerstört hatte. (

Das Bitten wird in (1) als Prozess dargestellt, dessen Grenze nicht präfixal (wie sonst für die Slavia üblich) indiziert wird, auch wenn sie mitgedacht wird. Auch wenn die Reise in (2) als abgeschlossen dargestellt wird, ändert dies natürlich nichts an der Tatsache, dass das Verb 'reisen' ein Durativum tantum ist.

In den Sprachen, wo es keine Opposition von Imperfekt und Aorist gibt, wird die jeweilige Lesart bei den atelischen Verben jeweils kontextgesteuert, vgl.

Deutsch:

(3) Elke hat gestern ihre Unterlagen lange gesucht. [imperfektiv-durativ]. vs.

(4) Elke hat ihre Unterlagen gestern schon gesucht [,,aoristisch“-komplexiv], sodass sie sie heute nicht wieder suchen will. 
Polnisch:

(5) Elke wczoraj długo szukała swoich dokumentów.

(6) Elke wczoraj już szukala swoich dokumentów, więc nie chce szukać ich dziś ponownie.

In den Beispielen mit der jeweils geraden Nummer handelt es sich um die Außenperspektivierung von Ereignissen, welche sonst dank ihrer atelischen Aktionsartsemantik in unmarkierten Kontexten innenperspektivisch gelesen werden. Diese Opposition wurde in den Sprachen mit Imperfekt und Aorist entsprechend morphologisch markiert, auch wenn die Aktionsartsemantik der Verben unverändert blieb, wodurch die „lexikalische“ und die ,viewpoint“"-Aspektualität getrennt kodiert wurden.

In den modernen ost- und westslawischen Sprachen, die die Aoristkategorie verloren haben, werden nun potentiell ,aoristische“ Lesarten regulär an die atelischen Verben gebunden, während die telischen Verben lediglich die perfektiven Lesarten beibehalten und nur im derivationell kodierten perfektiven Aspekt erscheinen können, bei dem das jeweilige Affix sowohl Aktionsart (Telizität) als auch Aspekt (Perfektiv) ausdrückt, vgl.

Polnisch:

(7) Norbert siedział w swoim pokoju i czytał gazetę. [imperfektiv und atelisch] Innenperspektive

(8) Norbert już czytal tę gazetę. [formal imperfektiv, funktional komplexiv] Außenperspektive

(9) Norbert przeczytal gazetę i poszedł do sypialni. [perfektiv und telisch] Außenperspektive

Die komplexive Lesart neutralisiert die binäre privative Opposition von Imperfektiv und Perfektiv zu Gunsten des merkmallosen Gliedes, der imperfektiven Aspektform, die jedoch eine deutlich außenperspektivische Lesart suggeriert. Dies ist aus sprachhistorischer Sicht die übliche Entwicklung bei allen Aspektsprachen, die die Aoristkategorie für die Indizierung einer Reihe von Aspektfunktionen in der Vergangenheitsperspektive einbüßen. Im Weiteren wird nun gezeigt, wie diese Funktionen im Sprachkontrast dargestellt werden können.

\section{Fallbeispiele im Kontrast}

Erklären wir mit Leiss (1992; 2002) die Innen- vs. Außenperspektivierung des Verbalgeschehens zum Hauptkriterium der aspektualen Lesarten auf Satzebene, müssen wir davon ausgehen, dass der sog. ,,viewpoint aspect“" im Prinzip auch in den Sprachen ohne grammatikalisierte Aspektkategorie vorhanden sein kann, allerdings als Eigenschaft von Strukturen mit beschränktem Grad an Paradigmatisierung und folglich auch ohne Systemzwang, der für Aspektsprachen typisch ist. Nichtsdestoweniger kann vermutet werden, dass die ,viewpoint“-Oppositionen nicht nur als overte, sondern vielmehr gerade als koverte kategoriale Signale in den Sprachen ohne indizierte Aspektkategorie feststellbar sind. Nun ist die Aufdeckung versteckter, „kryptotypischer“ Kategorial- 
funktionen eine der wichtigsten Aufgaben der linguistischen Theorie und eine echte Herausforderung an die Linguistik schlechthin. Von Stechow (1993: 1f.) vergleicht dieses Vorgehen mit der Aufdeckung der chemischen Formeln oder der Gene, die jeweils hinter „overten“ Stoffen resp. Blütenfarben stehen.

Wir können nun versuchen, ein Modell aufzubauen, in dem die Aktionsarten der Verben mit dem ,viewpoint“-Aspekt interagieren, und zwar unter der Maßgabe, dass die jeweilige Aktionsart sich nicht nur, wie es in der Literatur stets wiederholt wird, vom Aspekt unterscheidet, sondern gewisse „kategoriale Spuren“ des „,viewpoint“-Aspekts enthält. Für die slawischen Sprachen muss dies nicht extra nachgewiesen werden, da es eine empirisch leicht nachweisbare Evidenz ist: Jede Aktionsart ist entweder an den imperfektiven oder an den perfektiven Aspekt sozusagen ,automatisch“ gebunden. So sind z. B. die Durativa und Iterativa immer imperfektiv, während die Resultativa oder Inchoativa immer perfektiv sind. In anderen Aspektsprachen ist dies, wie oben bereits erwähnt, nicht der Fall, da dort die Verteilung der Aktionsarten auf die Aspektkategorie anders aussieht, wie dies oben am Beispiel des Griechischen oder des Altkirchenslawischen gezeigt wurde.

Eine gute Vergleichsbasis kann uns hier das moderne Englisch liefern. Einerseits sind seine Tempusformen weitestgehend ,aspektvermittelt" und andererseits hat es ein eigenes Aspektsystem als grammatikalisierte Opposition zwischen den Simple- und den Progressive-Formen, wobei die Letzteren deutlich markiert sind und die Funktion einer ,aktuellen Durativität“ (Smirnickaja 1977: 21) erfüllen, was in etwa der Grundfunktion des slawischen imperfektiven Aspekts entspricht, ohne dass dadurch andere Aktionsartfunktionen der slawischen Imperfektiva erfasst werden. Dennoch ist auch die englische Progressive-Form nicht nur aspektual, sondern auch temporal zu deuten.

Die Formen des englischen Simple Past Tense stehen denen des Present Perfect nicht nur rein temporal, sondern auch quasi ,aspektual“ gegenüber, auch wenn sie in den englischen Grammatikbüchern als Tempusformen behandelt werden. Davon zeugt die einfache Probe, die hier der Einfachheit halber als ,yesterday - for/already“-Test bezeichnet wird. Das Adverb der Zeitstufe Vergangenheit und alle damit vergleichbaren Indikatoren erfordern das Simple Past und schließen das Present Perfect aus, während die Präposition for, das Adverb already und damit vergleichbare Indikatoren umgekehrt das Simple Past ausschließen und das Present Perfect erfordern, vgl.

Englisch:

(10) Yesterday John wrote letters. vs.

(11) John has written letters for two days.

(11a) John has already written all the letters.

Natürlich wird hier durch die Form des Present Perfect ein temporal fassbarer Bezug der vergangenen Handlung zur Gegenwartsstufe kodiert, was generell außerhalb der 
Aspekt- oder Aktionsartfunktion liegt und rein tempusdeiktische Interpretation zulässt. Zugleich wird aber auch die Handlung aus der Perspektive der „viewpoint"-Aspektfunktion jeweils anders gedeutet. In (10) handelt es sich nämlich um eine vergangene Handlung, deren Grenzbezug nicht explizit ausgedrückt wird, weil er offensichtlich nicht signifikant ist. Es wird lediglich darauf hingewiesen, dass John eine gewisse Aktivität in der Vergangenheit erfüllte, dass also der gestrige Tag für ihn mit dem Briefe-Schreiben zumindest teilweise ausgefüllt war. In (11) geht es dagegen um ein Zeitintervall, das John für das Briefe-Schreiben benutzen musste, um seine Tätigkeit mehr oder minder sukzessiv abzuschließen. Der Blickpunkt der Origo ist also bei (10) innenperspektivisch: Es wird nämlich erzählt, womit sich John gestern befasst hat. Bei (11) und (11a) ist die Sicht dagegen außenperspektivisch: Es wird festgestellt, dass eine gewisse Tätigkeit von deren Anfangs- bis deren Endpunkt eine gewisse Zeit gedauert hat und danach abgeschlossen wurde. Die ,interne Zeit“ ist daher im ersten Fall „offen“ und im letzteren ,geschlossen“.

Wenn nun statt der Simple-Formen die Formen des Progressives eingesetzt werden, erhalten wir folgende Beispiele:

(12) Yesterday John was writing letters (,when a colleague of him entered his room).

(13) John has been writing letters for two days.

In (12) wird die innenperspektivische Sicht nicht nur einfach in der Vergangenheit situiert, wie in (10), sondern auf einen bestimmten Zeitpunkt bezogen, in dem die andauernde Handlung gerade in ihrem Verlauf fixiert wird und unter keinen Umständen als abgeschlossen gelten kann (,aktuelle Durativität“ nach Smirnickaja, siehe oben). Die Handlung in (13) ist zwar abgeschlossen, aber es wird - im Unterschied zu (11a) betont, dass sie eine Zeit lang gedauert hat. Deshalb ist hier die Verwendung des strikt „begrenzenden“ Adverbs already blockiert. Diese Perspektivierung ist nun doppelt zu fassen: Der Abschluss der Handlung versetzt die Origo in die Situation der „Außenbeobachterin“, während ihre Dauer eher die „Innenperspektivierung“ favorisiert. An diesem letzteren Fall kann gut beobachtet werden, dass in einigen Sprachsystemen der „lexikalische“ Aspekt (die Opposition der Merkmale telisch vs. atelisch) nicht mit dem „viewpoint“-Aspekt (die Opposition der Merkmale perfektiv vs. imperfektiv) einhergeht, anders also als in der Slavia. Das Beispiel (12) demonstriert Atelizität und gleichzeitig Imperfektivität, während (13) Atelizität mit Perfektivität verbindet.

Nun gehört das Verb write wie die Mehrheit anthropozentrischer und objektbezogener (transitiver) Verben nach der klassischen Klassifikation der aktionsartgestützten Verbalbedeutungen von Vendler (1957) zu den sogenannten ,accomplishments“, d. h. Verben einer potentiell abgeschlossenen Tätigkeit. Diese Verben drücken einerseits Handlungen aus und sind daher der Klasse der sogenannten ,activities“ ähnlich, aber im Unterschied zu ihnen kodieren sie nicht eine als unbegrenzt konzipierte Handlung (wie z. B. die Verben des Typs suchen, spielen, fahren u. a.). Deshalb können diese 
Verben prinzipiell sowohl telische als auch atelische Lesarten haben. Diese können allerdings von Sprache zu Sprache unterschiedlich bezeichnet werden. Wie oben gezeigt wurde, werden alle Lesarten im Englischen grammatisch overt markiert. Im Polnischen und Russischen, wo der ,viewpoint“-Aspekt sich grundsätzlich mit Telizität/Atelizität deckt, wird das jeweilige präfigierte (perfektive) Verb nur in telischer Aktionsartbedeutung verwendet, während die atelische Lesart vom unpräfigierten (imperfektiven) Verb kodiert wird:

Polnisch:

(14) Wczoraj John pisal listy.

(15) John napisal listy w ciągu dwóch dni.

(16) Wczoraj John pisal listy (, kiedy jego kolega wszedł do pokoju).

(17) John pisal listy dwa dni.

Die russischen Entsprechungen werden hier angesichts ihrer völligen Übereinstimmung mit den polnischen nicht angeführt.

Erklärungsbedürftig ist hier nur das Beispiel (17). Im entsprechenden englischen Beispiel (13) kreuzen sich, wie oben bereits gezeigt wurde, die äußerlich kontradiktorischen Merkmale der Atelizität und Perfektivität, von denen jedes durch ein spezifisches grammatisches Zeichen kodiert wird. Dies weist eine eindeutige Parallelität zu den unter (1) und (2) behandelten altkirchenslawischen resp. griechischen Belegen auf. Auf dieser Folie ist nun gut zu sehen, dass die in (17) kodierte Kategorialfunktion keine einfache „Summe“ von Atelizität und Perfektivität ist, sondern einer anderen Begrifflichkeit bedarf, um beide - augenscheinlich konträren - Funktionen auf einen gemeinsamen Nenner zu bringen. Die in (13) denotierte Bedeutung ist nämlich deutlich zur oben bereits behandelten Funktion des „komplexiven“ oder „konfektiven“ Aorists affin. Da das Englische hierzu zwei getrennte grammatische Signale besitzt, kann diese Funktion gut beobachtet werden. Die slawischen Sprachen realisieren die Bedeutung des „,komplexiven“ bzw. „konfektiven“ Aorists, welcher als grammatische Kategorie im Laufe der Sprachgeschichte verloren gegangen ist, durch den üblichen Mechanismus der Neutralisierung der binären privativen Opposition zu Gunsten des jeweils unmarkierten (merkmallosen) Gliedes. Deshalb wird die komplexive Aktionsart durch den merkmallosen imperfektiven Aspekt ausgedrückt, der kontextbezogen diese Lesart „profiliert“.

In der deutschen Sprache, wo der grammatische Verbalaspekt fehlt, werden die Aktionsartlesarten grundsätzlich anders gesteuert. Im Prinzip können alle angeführten Beispielsätze im Deutschen sowohl im Präteritum als auch im Perfekt erscheinen. Der kategoriale Unterschied ist dort nicht aspektualer, sondern temporaler Natur, auch wenn das Präteritum im vergangenheitsbezogenen Narrativ im Unterschied zum „dialogischen“, aktuellen Perfekt erscheint (vgl. Darski 2015: 420f.). Nichtsdestoweniger kann die Opposition von „Innenperspektivierung“ und „Außenperspektivierung“ nicht an der Achse Präteritum : Perfekt beobachtet werden, vgl.: 
(18) Gestern schrieb John Briefe. / Gestern hat John Briefe geschrieben.

(19) John schrieb die Briefe innerhalb von zwei Tagen. / John hat die Briefe innerhalb von zwei Tagen geschrieben.

(20) Gestern schrieb John Briefe. / Gestern hat John Briefe geschrieben (, als sein Kollege in sein Zimmer eintrat / eingetreten ist).

(21) John schrieb Briefe zwei Tage lang. / John hat zwei Tage lang Briefe geschrieben.

Das einzige Indiz für Außenperspektivierung (,viewpoint“-Aspekt) und damit verbundene Telizität (,lexikalischer“ Aspekt) ist die Verwendung des bestimmten Artikels im Beispiel (19) (vgl. Leiss 2000; 2002: 47ff.). Bei der Kodierung ,perfektivischer Atelizität" (21) wird dagegen statt Artikelhaftigkeit Artikellosigkeit benutzt.

Bei den Verben des Typs 'schreiben', d. h. den ,potentiellen accomplishments“, sind kraft ihrer Aktionsartsemantik prinzipiell sowohl telisch-perfektive als auch atelisch-imperfektive bzw. komplexive/konfektive (atelisch-perfektive) Lesarten möglich. Bei den Verben anderer „Vendler'scher“ Aktionsartklassen sieht die Korrelation zwischen dem „viewpoint“-Aspekt und dem „lexikalischen“ Aspekt etwas anders aus.

Die sogenannten „states“ (atelische Prozess- und Zustandsverben) des Typs rollen, liegen, sitzen, schlafen u. a. und ,activities“ (atelische Tätigkeitsverben) des Typs suchen, rennen, spielen, arbeiten verhalten sich wie folgt: Atelizität ist ihnen immanent, sodass sie in keinem Kontext telisch gelesen werden können. Dies heißt jedoch nicht, dass sie zugleich stets imperfektivisch verwendet werden, auch wenn die imperfektivische Lesart für sie ohne Zweifel stets merkmallos ist. Die „Außenperspektivierung“ schafft hier zwar keine perfektivischen Lesarten, aber sie ist durchaus mit Komplexivität/Konfektivität affin, welche in den Sprachen mit der Aoristkategorie, aber auch z. B. im Englischen overt und in der Slavia und im Deutschen kovert kodiert wird, vgl.

Englisch:

(22) Yesterday John slept quietly / played with his sister.

(23) John was sleeping / playing with his sister (, when I entered the room).

(24) John has slept/played with his sister for two hours.

(25) John has been sleeping / playing with his sister for two hours.

(26) Polnisch (ähnlich Russisch):

(27) Wczoraj John spal spokojnie / bawil się ze swoją siostrą.

(28) John spal / bawil się z siostrą (, kiedy ja wszedłem do pokoju).

(29) John spal / bawil się z siostra dwie godziny.

(30) John spal / bawil się z siostrą w ciągu dwóch godzin.

Deutsch:

(31) Gestern schlief John ruhig / hat John ruhig geschlafen / spielte John mit seiner Schwester / hat John mit seiner Schwester gespielt.

(32) John schlief / hat geschlafen / spielte mit seiner Schwester / hat mit seiner Schwester gespielt (, als ich ins Zimmer eintrat / eingetreten bin). 
(33) John hat zwei Stunden geschlafen / mit seiner Schwester gespielt.

(34) John hat zwei Stunden lang geschlafen / mit seiner Schwester gespielt.

Die komplexive Lesart - jeweils in (25), (29) und (33) - wird im Englischen durch die grammatische Form des Present Perfect Progressive ausgedrückt, während sie im Deutschen und im Polnischen lediglich mithilfe eines Adverbs zusätzlich kodiert werden kann. Im Polnischen (wie auch im Russischen) werden dabei - in Einklang mit der Faustregel der Aspekt-Aktionsart-Korrelation - das ,state“-Verb sowie das ,,activity“-Verb nur im imperfektiven Aspekt verwendet, auch wenn sich die „viewpoint"-Lesart ändert. Freilich kann hier Telizität in (27) und (29) sekundär durch ein Präfix (poln., russ. po-) indiziert werden (pospat, pobawit się), aber in diesem Fall erhält man keine „reine“ Telizität, sondern stets eine zusätzliche Aktionsartbedeutung (Perdurativität bzw. Delimitativität), die streng gesagt die lexikalische Semantik des Grundverbs verändert, etwa neben Intervallbegrenzung die relative Kürze des Intervalls oder aber die 'durch'-Semantik involviert (ausführlicher hierzu vgl. Nübler 1992; Kątny 1994; Czarnecki 1998). Das Vorhandensein der Perdurativa in der Slavia betrachtet Nübler (1992: 37) als Beweis für Insuffizienz der Vendler'schen Klassifizierung von Aktionsartverben, da sich diese Verben angeblich nicht in das Schema von Vendler einordnen lassen. In der Wirklichkeit handelt es sich jedoch bei diesen und mehreren anderen „abgeleiteten“ Aktionsartklassen um recht gut vorhersagbare Modifizierungen der Vendler'schen „Grundklassen“ in den Sprachsystemen mit indiziertem Verbalaspekt und derivationell bildbaren Aktionsarten, von denen jede unter nur einen Verbalaspekt fällt.

Bei den ,achievements“ (d. h. telischen intransitiven Perfektiva mit atomar-transformativer Semantik) des Typs einschlafen, geschehen, fallen, sterben sieht das Verhältnis zwischen lexikalischer und „viewpoint“-Aspektualität grundsätzlich anders aus als bei den drei oben behandelten Aktionsartklassen. Aus der Sicht der Opposition zwischen Telizität und Atelizität sind dies eindeutig telische Verben. Das bedeutet jedoch nicht, dass hiermit der Zwang entsteht, Telizität immer an Perfektivität zu binden. Freilich wird diese Bindung unter neutralen Kontextbedingungen in aller Regel impliziert, aber eine sekundäre „Imperfektivierung“ durch die Verschiebung der Blickrichtung der Origo auf die „Innenperspektive“ ist nicht ausgeschlossen, vgl.:

Englisch:

(35) John died yesterday at six.

(36) John was dying (, when his brother entered his room).

(37) John has already died.

(38) John has been dying for two hours.

Im Unterschied zu den accomplishments sind hierbei die Belege mit dem Present Perfect und mit dem Present Perfect Progressive aus der Sicht der Aspektbedeutung nicht nur „nuanciert“, sondern grundsätzlich unterschiedlich. Da eine atomare Trans- 
formation normalerweise keine in der Zeit gedehnte Lesart zulässt, wird in (36) nicht die Präposition for, sondern vielmehr das Adverb already selegiert, welches nur mit Außenperspektivierung des Verbalgeschehens kompatibel ist. In (35) haben wir es dagegen - trotz Telizität! - mit Innenperspektivierung zu tun, welche die allgemeine narrative Kodierung widerspiegelt: Über das Ereignis wird erzählt oder berichtet, wohingegen in (37) die typische außenperspektivische Bedeutung der achievements (Resultativität) kodiert wird, etwa 'John ist nun tot'.

In (36) und (38) wird durch die grammatische Progressive-Form Telizität als Prozess dargestellt, welcher auf ein Resultat gerichtet ist, aber dieses erst innerhalb eines Zeitintervalls erreicht. In (36) ist der Prozess „offen“ und deutlich aus der Innenperspektive der Origo dargestellt, wodurch im Prinzip das Resultat zwar zu erwarten, aber nicht zwingend vorauszusetzen ist. Telizität ist hiermit mit Imperfektivität affin. In (38) ist die Kategorialfunktion der grammatischen Form des achievement-Verbs äußerst komplex. Sowohl der lexikalische als auch der ,viewpoint“-Aspekt sind respektive telisch und perfektiv. Die Blickrichtung der Origo ist daher als außenperspektivisch zu behandeln. Trotzdem unterscheidet sich die Außenperspektive in (37) von der Außenperspektive in (38). Im ersten Fall handelt es sich um eine resultative Bedeutung, die nach ihrer Aktionsart-Semantik der Bedeutung eines „Resultatszustands“ nahe liegt. Im letzteren Fall dagegen wird innerhalb der Außenperspektive die interne „Teilung" des Zeitintervalls mit kodiert. Dies ist wiederum ein typisches Merkmal des komplexiven oder konfektiven Aorists.

In der Slavia haben die meisten achievements imperfektive Aspektpaare, welche nach dem Grundsatz des „Aspekts slawischen Typs“ innerhalb der übergreifenden „kategorialen Prozedur“ der sekundären (suffixal gesteuerten) Imperfektivierung von Perfektiva entstanden sind, vgl. poln. (ähnlich russ.): zasnacc' 'einschafen' : zasypiać' 'am Einschlafen sein', zdarzyć się '(einmal) geschehen' : zdarzać się 'mehrfach, oft, selten u. a. geschehen; vorkommen', umrzeć 'sterben' : umierać 'im Sterben liegen' u. a. Bereits aus diesen Auswahlbeispielen ist gut ersichtlich, dass die sekundäre Imperfektivierung der achievements unterschiedliche Lesarten bezüglich der jeweiligen Aktionsart ergibt, wenngleich der imperfektive (innenperspektivische), ,viewpoint"“-Aspekt in allen Fällen erhalten bleibt. Bei einigen Verben ist eine „Dehnung in der Zeit“ möglich, was eine durative Deutung involvieren kann, vgl. zasnaćc' 'einschlafen' : zasypiać' 'am Einschlafen sein', umrzeć 'sterben' : umierać 'im Sterben liegen'. Bei anderen Verben derselben Aktionsartklasse, wie z. B. zdarzyć się 'geschehen', znaleźć 'finden' u. a. ist die sekundäre Imperfektivierung grundsätzlich inkompatibel mit durativer Aktionsartfunktion, wodurch die ,nächstliegende“ Aktionsartbedeutung der Iterativität indiziert wird. Sie bezeichnet nicht ein additives Geschehen, das in jeder seiner Zeitphasen als weitgehend monoton und unverändert konzipiert wird, sondern die Wiederholung gleichartiger Geschehen, von denen jedes abgeschlossen ist, die aber in ihrer Aufeinanderfolge als monoton und additiv gedeutet werden: $z$ darzać się 'vorkommen', znajdować 'mehrmals finden' u. a. Übrigens lassen sich sekundär-durative Lesarten kontextbedingt auch iterativ deuten, etwa: 
(39) John zawsze zasypiat o pierwszej w nocy. 'John schlief immer um ein Uhr nachts ein.'

Mit durativer Umdeutung des telischen Verbalgeschehens der achievements sehen die Verwendungen der Aspektpaare wie folgt aus:

(40) John umarł wczoraj o szóstej.

(41) John właśnie umieral (, kiedy jego brat wszedł do pokoju).

(42) John już umarl (=nie żyje).

(43) John umieral dwie godziny.

Das Beispiel (43) enthält wiederum die komplexiv-aoristische Lesart: Einerseits wird das Ergebnis kodiert, andererseits ist das Zeitintervall mit eingeschlossen. Diese „doppelte Perspektivierung“ kann in einer Aspektsprache „slawischen Typs“ allerdings nur durch das unmarkierte Glied der binären privativen Opposition von Imperfektivität : Perfektivität, d. h. durch das imperfektive Verb, kodiert werden.

Im Deutschen kann von achievements keine „reguläre“ Imperfektivform gebildet werden, was angesichts des Fehlens des grammatischen Aspekts verständlich ist. Die achievements sind daher im Deutschen telische Verben und somit Perfectiva tantum. Der innenperspektivische ,viewpoint“-Aspekt kann hier allerdings durch spezifische, genuin lexikalisch-grammatische „Streckformen“ des Typs am+substantivierter Infinitiv + sein (vgl. u. v. a. Thieroff 1992; Abraham 2015), im+substantivierter Infinitiv+liegen $\mathrm{u}$. a. kodiert werden:

(44) John starb gestern um sechs Uhr abends / ist gestern um sechs Uhr abends gestorben.

(45) John lag im Sterben (, als sein Bruder das Zimmer betrat).

(46) John ist schon / nach zwei Stunden gestorben.

(47) *John starb zwei Stunden lang / *ist zwei Stunden lang gestorben.

Die komplexive Lesart ist also im Deutschen nicht möglich. Dies ergibt sich aus der ausschließlich telischen Semantik der achievements in Verbindung mit dem Fehlen des grammatischen Aspekts. Iterativität wird allerdings von dieser Restriktion ausgeschlossen, da Telizität sich als eine monotone Wiederholung abgeschlossener Ereignisse durchaus interpretieren lässt, vgl.:

(48) John schlief jeden Tag um ein Uhr nachts ein / ist jeden Tag um ein Uhr nachts eingeschlafen.

(49) Mehrmals verlor John seine Brieftasche, fand sie aber jedes Mal wieder.

Bezüglich der deutschen Konstruktion am+substantivierter Infinitiv+sein, die in der aktuellen Forschung intensiv untersucht wird, kann hier nur gesagt werden, dass sie im Gegenwartsdeutschen nicht nur mit den achievements, sondern auch mit den Verben anderer Aktionsartklassen gebildet werden kann: Er ist am Lesen, am Briefe-Schreiben, am Spielen u. a. Natürlich hat sie dort andere Funktionen als „Dehnung“ einer telischen Aktionsart, aber deren Erweiterung auf andere Aktionsartklassen zeugt 
eindeutig von einer weit fortgeschrittenen Grammatikalisierung dieser syntaktischen Fügung im Bereich der imperfektiven Aspektualität.

\section{Versuch einer Gesamtinterpretation}

Die kategoriale Wechselwirkung von Aspekt- und Aktionsartfunktionen unterscheidet sich von Sprache zu Sprache. In der vorliegenden Studie wurden Sprachsysteme konfrontiert, in denen dieselben oder vergleichbare Funktionen overt oder aber kovert indiziert werden. Im Mittelpunkt des Interesses stand dabei die Interaktion zwischen dem „lexikalischen“ Aspekt (Opposition atelisch : telisch) und dem „viewpoint“-Aspekt (Opposition imperfektiv : perfektiv) und die Verteilung der jeweiligen Oppositionsglieder auf grammatische Tempus- und Aspektformen.

Es konnte festgestellt werden, dass neben der Grundopposition von innenperspektivisch konzipiertem Imperfektivum und außenperspektivisch konzipiertem Perfektivum andere ,viewpoint“-Oppositionen existieren, von denen die Kodierung der komplexiven Funktion in der Vergangenheitsperspektive besonders interessant erscheint. Diese Funktion war in den Sprachen, die die Aorist-Kategorie enthielten, eine der mannigfaltigen Funktionen des Aorists. Der komplexive oder damit vergleichbare konfektive Aorist war u. a. im Altgriechischen oder im Altkirchenslawischen vorhanden. Seine Funktion kann als doppelter Bezug auf Abschluss des Verbalgeschehens und zugleich auf das Zeitintervall, in dem es dauerte, beschrieben werden.

Unter dieser Maßgabe wird klar, dass die konkrete Deutung der Komplexivität je nach der Aktionsartsemantik des entsprechenden Verbs unterschiedlich ist. Daher ist in dieser Studie eine getrennte Interpretation von Beispielen mit den Verben der klassischen, Vendler'schen Aktionsarttypen vorgenommen worden, den sogenannten states, activities, accomplishments und achievements. Es ist evident, dass bei den Verben, die den Prozessbezug durch ihre Semantik betonen, potentiell implizieren oder ausschließen, das Merkmal der Komplexivität unterschiedlich gedeutet wird, wodurch der aspektuale „viewpoint“ jeweils anders modifiziert wird.

Die kontrastive Analyse hat nun ergeben, dass das Englische aus der hier behandelten Perspektive die „stärksten“ grammatischen Signale besitzt, da es (i) aspektsensitive Tempusformen Past und Present Perfect und (ii) den grammatisch indizierten progressiven Aspekt besitzt. Ihre Interaktion ergibt eine grammatisch gesteuerte Konfiguration des imperfektiven, des perfektiven und des komplexiven „,viewpoints“. Die grammatische Form des englischen Present Perfect Progressive kann dabei als nahezu vollidentisch mit dem „klassischen“ komplexiven oder auch konfektiven Aorist eingestuft werden. Somit kann für das Englische die grammatische Form des komplexiven/ konfektiven Aorists postuliert werden.

Die slawischen Sprachen steuern die entsprechenden Kategorialfunktionen durch die Aspektkategorie, welche die strikte Binarität von Perfektivität und Imperfektivität 
kodiert, wobei die aktionsartgesteuerte Opposition von Telizität und Atelizität damit engst verzahnt ist. Die komplexive Semantik wird dabei formal durch imperfektive Verben bezeichnet, welche jedoch in diesem Fall eine andere, außenperspektivische Deutung bekommen. Dies ist damit verbunden, dass im grammatischen Aspektpaar in der Slavia jeweils das imperfektive Glied der binären privativen Opposition merkmallos ist und daher die Neutralisierung derselben zu Gunsten der Imperfektivität erfolgt, auch wenn sie anders als klassische, kanonische (durativ gedeutete) Aspektsemantik gelesen wird. Dadurch bleibt die Formenopposition strikt binär (imperfektiv vs. perfektiv), während die funktionale Opposition ternär ist. Die perfektiven Verben drücken telische Perfektivität aus, während die imperfektiven Verben atelische Imperfektivität oder ,aoristische“ Komplexivität kodieren.

Das aspektlose Deutsch steuert sämtliche kategorialen Oppositionen durch die Aktionsartsemantik der jeweiligen Verben, greift aber auch zu anderen Mitteln, u. a. Artikel in der Nominalphrase oder spezielle imperfektivierende syntaktische Formen (am-Progressiv), um jeweils die außenperspektivische bzw. innenperspektivische Sicht zu indizieren und somit die Aspektfunktion kovert zu kodieren. Nichtsdestoweniger bleiben im Deutschen einige „Lücken“ unausgefüllt, vor allem die komplexive Lesart bei achievements.

\section{Literatur}

Abraham, Werner (2015): "Predicative copula + infinitive forms and their diverse functions in German". In: Kotin, Michail L., in collaboration with Richard J. Whitt (Hg.): To be or not to be? The Verbum Substantivum from Synchronic, Diachronic and Typological Perspectives. Newcastle upon Tyne: Cambridge Scholar Publishing. 94-122.

Abraham, Werner/Janssen, Theo (Hgg.) (1989): Tempus - Aspekt - Modus: Die lexikalischen und grammatischen Formen in den germanischen Sprachen. Tübingen: Max Niemeyer Verlag.

Abraham, Werner/Leiss, Elisabeth (2012): Covert Patterns of Modality. Newcastle upon Tyne: Cambridge Scholar Publishers.

Bondarko, Aleksandr V. (1995): Die Semantik des Verbalaspekts im Russischen. Frankfurt am Main: Peter Lang Verlag.

Bybee, Joan/Dahl, Östen (1989): „The creation of tense and aspect systems in the languages of the world“. Studies in Language 13 (1), 51-103.

Comrie, Bernard (1976): Aspect. An introduction to the study of verbal aspect and related problems. Cambridge: Cambridge University Press.

Czarnecki, Tomasz (1998): Aspektualität im Polnischen und im Deutschen. Bedeutungen und Formen in einer konfrontativen Übersicht. Gdańsk: Wydawnictwo Uniwersytetu Gdańskiego.

Dahl, Östen (1985): Tense and Aspect Systems. Oxford: Blackwell.

Darski, Józef Paweł (1999): Bildung der Verbformen im Standarddeutschen. Tübingen: Stauffenburg.

Darski, Józef Paweł (2004): Linguistisches Analysemodell. Definitionen grundlegender grammatischer Begriffe. 2., völlig neu bearbeitete und ergänzte Aufl. Poznań: Wydawnictwo Naukowe UAM.

Darski, Józef Paweł (2010): Deutsche Grammatik. Ein völlig neuer Ansatz. Frankfurt a. M. u. a.: Peter Lang Verlag. 
Darski, Józef Paweł (2015): Gramatyka niemiecka z uwagami konfrontatywnymi. Wydanie II, poprawione i rozszerzone. Poznań: Wydawnictwo Naukowe UAM.

Dostál, Antonin (1954): Studie o vidiovém systému v staroslověnštině. Prague: Státni pedagogocké nakladetelství.

Eckhof, Hanne/Haug, Dag (2015): “Aspect and prefixation in Old Church Slavonic”. Diachronica 32/2, 186-230.

Fanning, Buist M. (1990): Verbal Aspekt in New Testament Greek. Oxford: Clarendon Press.

Forsyth, James (1972): "The nature and development of the aspectual opposition in the Russian verb". The Slavonic and East European Review 50 (1), 493-506.

Harris, Zellig Sabbetai (1951): Methods in Structural Linguistics. Chicago: University of Chicago Press.

Hjelmslev, Louise Troll (1943): Prolegomena to a Theory of Language. Baltimore: Indiana University Publications in Anthropology and Linguistics.

Isačenko, Aleksandr V. (1960): Grammatičeskij stroj russkogo jazyka v sopostavlenii s slovackim. Bratislava: Izdatel'stavo Slovackoj Akademii Nauk.

Jakobson, Roman (1932): „Zur Struktur des russischen Verbums“. In: Charisteria Guilelmo Methesio quinquagenario: a discipulis et Circuli linguistici Pragensis sodalibus oblate. Prague: Cercle Linguistique de Prague. 74-84.

Jarceva, Viktorija N. (1968): Vzaimootnošenija grammatiki i leksiki v sisteme jazyka. Moskau: Nauka.

Jones, Howard (2009): Aktionsart in the Old High German Passive. Hamburg: Buske.

Kątny, Andrzej (1994): Zu ausgewählten Aktionsarten im Polnischen und deren Entsprechungen im Deutschen. Rzeszów: Wydawnictwo Wyższej Szkoły Pedagogicznej.

Klein, Wolfgang (1994): Time in language. London: Routledge.

Klein, Wolfgang (1995): “A time-relational analysis of Russian aspect”. Language 71, 669-695.

Kotin, Michail L. (2008): „Das „Linguistische Analysemodell“ von Józef Darski im sprachhistorischen bzw. sprachtypologischen Diskurs“. In: Mikołajczyk, Beata/Michail L. Kotin (Hgg.): Terra grammatica. Ideen - Methoden - Modelle. Festschrift für Józef Darski zum 65. Geburtstag. Frankfurt a. M. u. a.: Peter Lang Verlag. 167-177.

Kotin, Michail L. (2012): Gotisch. Im (diachronischen und typologischen) Vergleich. Heidelberg: Universitätsverlag Winter.

Krasuchin, Konstantin G. (2007): „Aspekty i vremena praindojevropejskogo glagola. II. Aorist i perfekt drevnegrečeskogo glagola". Voprosy jazykoznanija 4, 8-36.

Lazorczyk, Agnieszka Agata (2010): Decomposing Slavic aspect: The role of aspectual morphology in Polish and other Slavic languages. Dissertation. Los Angeles: University of Southern California.

Leiss, Elisabeth (1992): Die Verbalkategorien des Deutschen. Ein Beitrag zur Theorie der sprachlichen Kategorisierung. Berlin/New York: Walter de Gruyter.

Leiss, Elisabeth (2000): Artikel und Aspekt: Die grammatischen Muster von Definitheit. Berlin/New York: Walter de Gruyter.

Leiss, Elisabeth (2002): (1) Die Rolle der Kategorie des Aspekts im Sprachwandel des Deutschen: ein Überblick; (2) Der Verlust der aspektuellen Verbpaare und seine Folgen im Bereich der Verbalkategorien des Deutschen; (3) Der Verlust der aspektuellen Verbpaare und seine Folgen im Bereich der Nominalkategorien des Deutschen. In: Japanische Gesellschaft für Germanistik (Hg.): Grammatische Kategorien aus sprachhistorischer und typologischer Perspektive. Akten des 29. Linguisten-Seminars (Kyoto, 2001). München: Iudicium Verlag, 9-58.

Maslov, Jurij S. (2004): „Rol’ tak nazyvajemoj perfektivacii i imperfektivacii v processe vozniknovenija slavjanskogo glagol'nogo vida“. In: Maslov, Jurij S. (Hg.): Izbrannyje trudy: Aspektologija, obščeje jazykoznanije. Moskau: Jazyki slavjanskoj kultury. 165-195.

Moulton, James H. (1906): A Grammar of New Testament Greek. Vol. 1: Prolegomena. $3^{\text {rd }}$ ed. Edinburgh: Clark. 
Nübler, Norbert (1992): „Perdurative und nichtdurative Verballexeme im Tschechischen und Russischen“. Studia Minora Fakultatis Philosophicae Universitatis Brunensis A 40, 33-42.

Schwall, Ulrike (1991): Aspektualität: Eine semantisch-funktionelle Kategorie. Tübingen: Gunter Narr Verlag.

Smirnickaja, Olga A. (1977): „Evolucija vido-vremennoj sistemy v germanskich jazykach“. In: Jarceva, Viktoria N. u. a. (Hgg.): Istoriko-tipologičeskaja morfologija germanskich jazykov. Bd. 2. Moskau: Nauka. 5-127.

Stechow, Arnim von (1993): „Die Aufgaben der Syntax“. In: Jacobs, Joachim/Stechow, Arnim von/Sternefeld, Wolfgang/Vennemann, Theo (Hgg.): Syntax. Ein internationales Handbuch zeitgenössischer Forschung. Berlin/New York: Walter de Gruyter. 1-88.

Streitberg, Wilhelm (1920): Gotisches Elementarbuch. 5. und 6. Aufl. Heidelberg: Winter.

Thieroff, Rolf(1992): Das finite Verb im Deutschen. Tempus - Modus - Distanz. Tübingen: Max Niemeyer Verlag.

Vendler, Zeno (1957): „Verbs and Times“. The Philosophical Review 66/2, 143-160.

Woźnicka, Marta (2014): „Zum Wandel in den Personal- und Numerusexponenten im Konjugationsparadigma vom Alt- bis zum Mittelhochdeutschen: Versuch einer Neuordnung aufgrund eines neuen Modells.“ In: Ágel, Vilmos/Gardt, Andreas (Hgg.): Paradigmen der Sprachgeschichtsschreibung. Berlin/Boston: Walter de Gruyter. 194-206. 